\title{
MICROWAVE ASSISTED SYNTHESIS OF BIOLOGICALLY ACTIVE 4-HYDROXY-N'-(PHENYLCARBONYL)- 2H-1,2-BENZOTHIAZINE-3-CARBOHYDRAZIDE 1,1-DIOXIDE DERIVATIVES
}

\author{
MUHAMMAD ZAHEER ${ }^{1,2}$, MUHAMMAD ZIA-UR-REHMAN ${ }^{1}$, SALMA RAHMAN ${ }^{1}$, NAVEED AHMED \\ AND MUHAMMAD NAWAZ CHAUDHARY2
}

\author{
${ }^{I}$ Applied Chemistry Research Centre, PCSIR Laboratories Complex, Ferozpur Road, Lahore-54600, Pakistan \\ ${ }^{2}$ Centre for Envoirnment \& Earth Sciences, University of the Punjab, Lahore-54590, Pakistan \\ ${ }^{3}$ Forman Christian College - A Chartered University, Canal Road, Lahore-54600, Pakistan
}

(Received: November 22, 2011 - Accepted: September 18, 2012)

\begin{abstract}
Microwave assisted synthesis of a series of 4-hydroxy- $N$ '-(phenylcarbonyl)-2H-1,2-benzothiazine-3-carbohydrazide 1,1-dioxides from sodium saccharin is reported. Sodium o-benzosulfimide was $N$-alkylated with ethyl chloroacetate followed by base catalyzed ring expansion to six membered 1,2-benzothiazine through Gabriel-Coleman type rearrangement yielding ethyl 4-hydroxy-2H-1,2-benzothiazine-3-carboxylate 1,1-dioxide. It was later condensed with a series of benzohydrazides synthesized from various carboxylic acids to get the title compounds. All the synthesized compounds were subjected to preliminary evaluation for their biological activity against series of Gram positive, Gram negative bacteria and fungi. Some of the compounds showed marked activity against the selected microorganisms.
\end{abstract}

Key Words: Microwaves; Benzohydrazide; Carbohydrazides; 1,2-Benzothiazine; Anti-bacterial; Anti-fungal.

\section{INTRODUCTION}

There is a need for development of clean chemical processes including synthetic procedures, catalysis and reaction conditions to reduce the environmental impact of chemical \& pharmaceutical industry. To achieve this, innovative and unconventional synthetic procedures using water as solvent ${ }^{1}$ under normal ${ }^{2}$ and super heated conditions ${ }^{3}$, in supercritical fluids ${ }^{4}$, ionic liquids ${ }^{5}$ and micro-emulsions ${ }^{6}$ are being used now a days. Besides these, ultrasound $\mathrm{d}^{7}$ and microwaves ${ }^{8}$ assisted reactions both in solvent as well as in solventless conditions are becoming popular due to their shorter reaction times and greater yields.

Hydrazides and related compounds are known as useful building blocks for the synthesis of various heterocyclic rings9. A large number of carbohydrazides and their derivatives have been reported in literature focusing their biological activities which include anti-fungal, anti-viral \& bacteriostatic ${ }^{11}$, anti-parasite ${ }^{12}$, anti-tuberculous ${ }^{13}$ and insecticidal ${ }^{14}$ activities. A large number of carbohydrazides and related compounds which are usually synthesized by hydrazinolysis of carboxylic acid esters are found to possess versatile activities. ${ }^{15,16}$

1,2-Benzothiazine 1,1-dioxide nucleus, firstly synthesized by Braun et $\mathrm{al}^{17}$ is familiar for its various derivatives like Sudoxicam $\mathbb{}$, Ampiroxicam ${ }^{\circledR}$, Meloxicam ${ }^{\circledR}$ and Lornoxicam ${ }^{\circledR}$. These are known as oxicams and are being used worldwide as non-steroidal anti-inflammatory drugs (NSAIDs) ${ }^{18}$ [Figure 1]. Benzothiazine derivatives are also familiar for their other biological activities e.g., 3,4-dihydro-1,2-benzothiazine-3-carboxylate 1,1-dioxide $\alpha$-ketomide and $\mathrm{P}(2)-\mathrm{P}(3)$ peptide mimetic aldehyde compounds are reported as potent calpain I inhibitors ${ }^{19}$ while 1,2-benzothiazin-3-yl-quinazolin-4( $3 H$ )-ones possess antibacterial properties ${ }^{20}$. They have also been found as anti-oxidants ${ }^{21-24}$ and for the treatment of rheumatoid arthritis, ankylosing spondylitis, osteoarthrosis and other inflammatory rheumatic and non-rheumatic processes ${ }^{25}$.

Keeping in view the potential biological activities of 1,2-benzothiazine1,1-dioxides and carbohydrazides, it was perceived that if both the heterocyclic moieties are synergized in a single nucleus, the newly obtained compounds may possess significant biological activities of individual compounds. In this quest, novel 4-hydroxy- $N^{\prime}$-(phenylcarbonyl)-2H-1,2-benzothiazine-3carbohydrazide 1,1-dioxides derivatives $\mathbf{( 8 a - 1 )}$ are synthesized by microwave assisted reactions of various benzohydrazides (7a-l) and ethyl 4-hydroxy- $2 \mathrm{H}$ 1,2-benzothiazine-3-carboxylate 1,1-dioxide (4) along with their preliminary assay for anti-bacterial and anti-fungal activities [Scheme 1].<smiles>CCCOC(=O)C1=C(O)c2ccccc2S(=O)(=O)N1C</smiles><smiles>COC(=O)C1=C(O)c2ccccc2S(=O)(=O)N1C</smiles><smiles>CN1C(C(=O)Nc2ccccn2)=C(O)c2sc(Cl)cc2S1(=O)=O</smiles><smiles>CN1C(C(=O)Nc2ccccn2)=C(O)c2sccc2S1(=O)=O</smiles>

Figure 1: Structures of few commercially available oxicam drugs

\section{EXPERIMENTAL}

\section{Chemistry:}

All the chemicals were purchased from E. Merck, BDH or Fluka and used without purification. However, solvents were purified through distillation. ${ }^{1} \mathrm{H}-$ NMR spectra were recorded on a Bruker DPX-400 instrument at $400 \mathrm{MHz}$. Chemical shifts are reported in ppm referenced to the residual solvent signal. FT-IR spectra were recorded on a Bruker Tensor 27 spectrometer. Mass spectra were recorded on Agilent $5973 \mathrm{~N}$ instrument using EI mode. Melting points were recorded on a Mettller Toledo FP 62 melting point apparatus and are uncorrected. Microwave assisted reactions were carried out in a household Microwave oven (Orient eNNe781JF) equipped with inverter technology (generating fixed frequency throughout the required time) for realistic control of the microwaves operating at multiples of 100 Watts up to 1000 Watts generating $2450 \mathrm{MHz}$ frequency. The apparatus was modified for laboratory applications, equipped with magnetic stirrer and an external reflux condenser.

Synthesis of ethyl (1,1-dioxido-3-oxo-1,2-benzisothiazol-2(3H)-yl)acetate (3)

A mixture of ethylchloroacetate $(1.19 \mathrm{~g}, 9.71 \mathrm{mmoles})$, sodium saccharin $(1.50 \mathrm{~g}, 7.31 \mathrm{mmoles})$ and dimethylformamide $(10 \mathrm{ml})$ was taken in a round bottom flask. The mixture was heated at water bath for 4 hours. The reaction mixture was cooled to room temperature and poured into ice cold water (60 $\mathrm{ml}$ ) to get white solid, which was filtered and washed with water. The solid was dried to get white crystalline solid mp $105-106^{\circ} \mathrm{C}$ (Lit. mp $\left.104-106^{\circ} \mathrm{C}\right) \cdot{ }^{17} \mathrm{IR}$ (KBr): 1752, 1743, 1340, $1181 \mathrm{~cm}^{-1} .{ }^{1} \mathrm{H}-\mathrm{NMR}\left(\mathrm{CDCl}_{3}\right), \delta: 1.29(\mathrm{t}, \mathrm{J}=7.2 \mathrm{~Hz}$, 


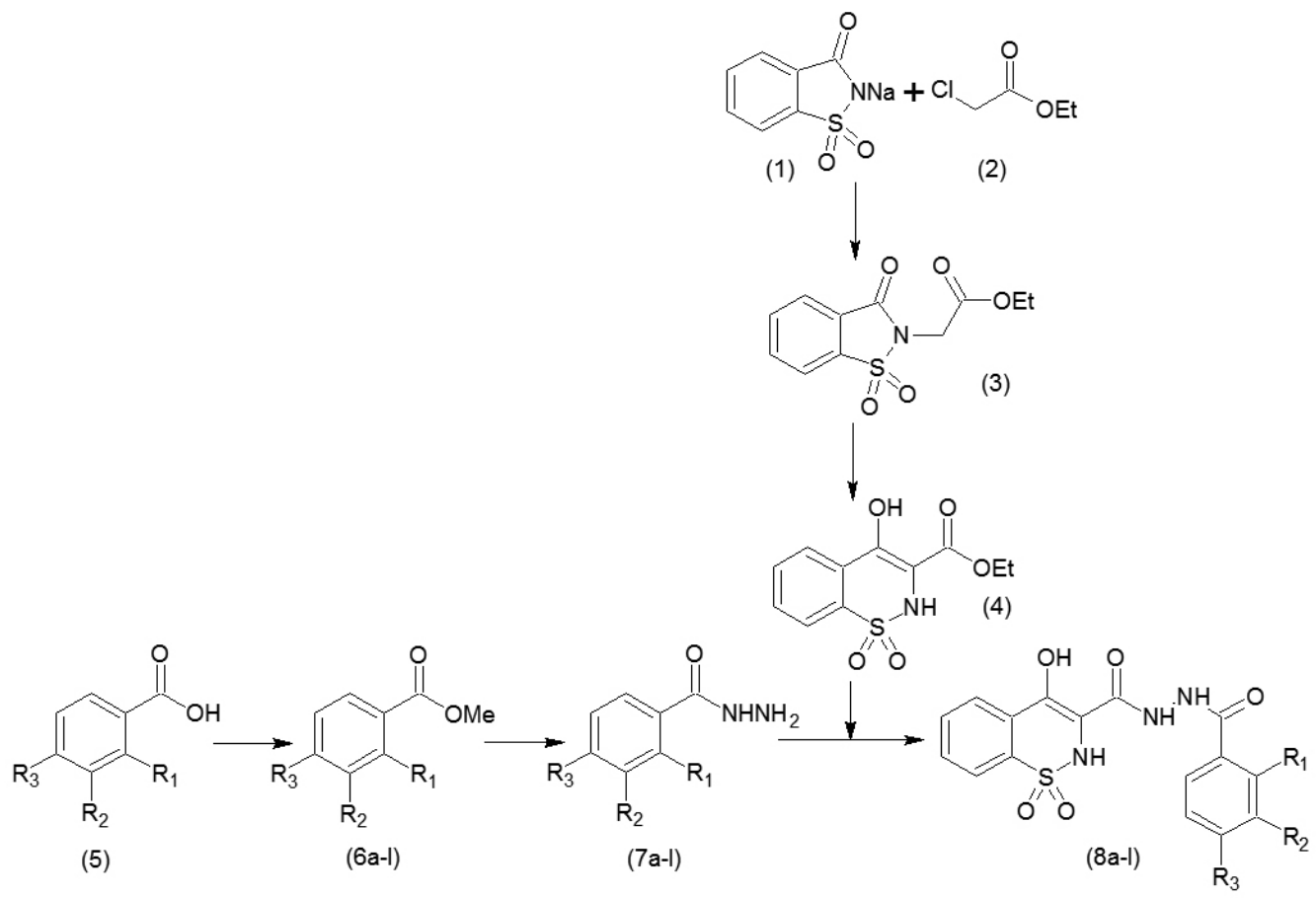

Scheme 1: Schematic synthesis of 4-hydroxy- $N$-(phenylcarbonyl)- $2 H$-1,2-benzothiazine-3-carbohydrazide 1,1-dioxides from carboxylic acids and sodium saccharin

$3 \mathrm{H}), 4.30(\mathrm{q}, \mathrm{J}=7.2 \mathrm{~Hz}, 2 \mathrm{H}), 4.41(\mathrm{~s}, 2 \mathrm{H}), 7.74(\mathrm{dd}, \mathrm{J}=5.6,3.2 \mathrm{~Hz}, 2 \mathrm{H}), 7.80$ (dd, J = 5.6, 3.2Hz, 2H). MS m/z: $269\left[\mathrm{M}^{+}\right], 224\left[\mathrm{M}^{+}-\mathrm{OC}_{2} \mathrm{H}_{5}\right]$. Anal. Calcd for $\mathrm{C}_{11} \mathrm{H}_{11} \mathrm{NO}_{5} \mathrm{~S}$ : C, 49.06; H, 4.12; N, 5.20; Found: C, 49.08; H, 4.11; N, 5.19. HRMS: Calcd. 269.0358, Found 269.0360.

Synthesis of ethyl 4-hydroxy-2H-1,2-benzothiazine-3-carboxylate 1,1-dioxide (4)

Sodium metal (2.7 g, 115 mmoles) was added to dry ethanol $(60 \mathrm{ml})$ in small portions. On the complete dissolution of sodium, the solution was concentrated in vacuum for the synthesis of sodium ethoxide suspension. To it ester (4) $(10.0 \mathrm{~g}, 37.17 \mathrm{mmol})$ and absolute ethanol $(30 \mathrm{ml})$ were added and sonicated for 4 minutes in ultrasonic bath at room temperature. Hydrochloric acid $(430 \mathrm{ml})$ was added to the reaction mixture to precipitate the product which was filtered and washed with water.

White crystalline solid; mp $138-139^{\circ} \mathrm{C}\left(\right.$ Lit. mp $\left.136-139{ }^{\circ} \mathrm{C}\right) .{ }^{17} \mathrm{IR}(\mathrm{KBr})$ : 3180, 1667, 1338, $1179 \mathrm{~cm}^{-1}$. ${ }^{1} \mathrm{H}-\mathrm{NMR}\left(\mathrm{CDCl}_{3}\right), \delta: 1.33(\mathrm{t}, \mathrm{J}=7.2 \mathrm{~Hz}, 3 \mathrm{H})$, 4.25 (q, J = 7.2 Hz, 2H), 6.42 (br s, NH), 7.74 (dd, J = 5.6, $3.2 \mathrm{~Hz}, 2 \mathrm{H}), 7.80$ $(\mathrm{dd}, \mathrm{J}=5.6,3.2 \mathrm{~Hz}, 2 \mathrm{H}), 12.38(\mathrm{~s}, 1 \mathrm{H}) . \mathrm{MS} \mathrm{m} / \mathrm{z}: 269\left[\mathrm{M}^{+}\right], 268\left[\mathrm{M}^{+}-\mathrm{H}\right], 224$ $\left[\mathrm{M}^{+}-\mathrm{OC}_{2} \mathrm{H}_{5}\right]$. HRMS: Calcd: 269.0358, Found: 269.0359. (6a-l)

General procedure for the synthesis of alkyl benzoates compounds

A mixture of substituted benzoic acid in methanol $(50 \mathrm{ml})$ and concentrated sulphuric acid (2-3 drops) was refluxed in microwave oven till the completion of reaction as indicated by TLC. After completion of reaction; excess methanol was distilled off and the content were poured in ice cold aqueous sodium bicarbonate $(4 \%)$. The product obtained was washed with ice cold water and dried in oven. Experimental details are given in Table 1. (7a-l)

General procedure for the synthesis of substituted benzohydrazides

A mixture of substituted alkyl benzoate (6a-1) ( $0.001 \mathrm{moles})$ and hydrazine hydrate $(0.001 \mathrm{moles})$ in ethanol $(50 \mathrm{ml})$ was refluxed in a microwave oven till the completion of reaction as indicated by TLC. After the completion of reaction; excess ethanol was distilled off and acidified with hydrochloric acid $(5 \%)$. The product obtained was washed with ice cold water and dried in oven. Experimental details are given in Table 1.

General procedure for the synthesis of derivatives of 4-hydroxy- $N$ '(phenylcarbonyl)-2H-1,2-benzothiazine-3-carbohydrazide 1,1-dioxides (8a-l)

A mixture of substituted benzohydrazide [7a-1] (0.001moles) and ethyl 4-hydroxy-2H-1,2-benzothiazine-3-carboxylate 1,1-dioxide (4) $(0.001$ moles; $0.269 \mathrm{~g}$ ) in ethanol $(50 \mathrm{ml})$ was refluxed in a microwave oven till the completion of reaction. After the completion of reaction, excess ethanol was distilled off and acidified with $\mathrm{HCl}(5 \%)$. The product obtained was washed with ice cold water and dried in oven.

4-Hydroxy- $N$ '-[(2-hydroxyphenyl)carbonyl]-2H-1,2-benzothiazine-3carbohydrazide 1,1-dioxide (8a)

Pale yellow powder; mp: $116^{\circ} \mathrm{C}$. IR $(\mathrm{KBr}) \mathrm{Cm}^{-1}: 3749,3653,3562$ $2365,1643,1348,1177,1069 .{ }^{1} \mathrm{H}$ NMR (DMSO- $\left.d_{6}\right)(400 \mathrm{MHz}) \delta: 3.43(1 \mathrm{H}$, s, SO NH) 6.90-6.97(4H, m, ArH), 7.75-7.89 (4H, m, ArH), $8.18(1 \mathrm{H}, \mathrm{s}$, $-\mathrm{OH}) 9.72(1 \mathrm{H}, \mathrm{s},-\mathrm{N} H), 9.94(1 \mathrm{H}, \mathrm{s},-\mathrm{N} H), 13.55(1 \mathrm{H}, \mathrm{s},-\mathrm{OH}) . \mathrm{MS} \mathrm{m} / \mathrm{z} 375.0\left[\mathrm{M}^{+}\right]$

4-Hydroxy- $N$ '-[(3-hydroxyphenyl)carbonyl]-2H-1,2-benzothiazine-3carbohydrazide 1,1-dioxide (8b)

Dirty yellow powder; mp: $150^{\circ} \mathrm{C}$. IR $(\mathrm{KBr}) \mathrm{Cm}^{-1}: 3760,3657,3568$, $2366,1642,1349,1180,1070 .{ }^{1} \mathrm{H}$ NMR (DMSO- $\left.d_{6}\right)(400 \mathrm{MHz}) \delta: 3.31(1 \mathrm{H}, \mathrm{s}$, $\left.\mathrm{SO}_{2} \mathrm{NH}\right), 6.96(1 \mathrm{H}, \mathrm{d}, J=7.6 \mathrm{~Hz}, \mathrm{ArH}), 7.12(1 \mathrm{H}, \mathrm{d}, J=8.0 \mathrm{~Hz}, \mathrm{ArH}), 7.25-$ $7.42(4 \mathrm{H}, \mathrm{m}, \mathrm{ArH}), 7.77(1 \mathrm{H}, \mathrm{s}, \mathrm{ArH}), 7.87(1 \mathrm{H}, \mathrm{d}, J=7.2 \mathrm{~Hz}, \mathrm{ArH}), 8.19(1 \mathrm{H}, \mathrm{s}$, $-\mathrm{OH}) 9.72(1 \mathrm{H}, \mathrm{s},-\mathrm{N} H), 9.94(1 \mathrm{H}, \mathrm{s},-\mathrm{N} H), 13.55(1 \mathrm{H}, \mathrm{s},-\mathrm{OH}) . \mathrm{MS} \mathrm{m} / \mathrm{z} 375.0\left[\mathrm{M}^{+}\right]$

4-Hydroxy- $N$ '-[(4-hydroxyphenyl)carbonyl]-2H-1,2-benzothiazine-3carbohydrazide 1,1-dioxide (8c)

Dirty yellow powder; $\mathrm{mp}: 185^{\circ} \mathrm{C}$. IR $(\mathrm{KBr}) \mathrm{Cm}^{-1}: 3753,3655,3568,2365$, $1648,1345,1189,1078 .{ }^{1} \mathrm{H}$ NMR (DMSO- $\left.d_{6}\right)(400 \mathrm{MHz}) \delta: 3.36\left(1 \mathrm{H}, \mathrm{s}, \mathrm{SO}_{2} \mathrm{~N} H\right.$ ), 6.92(2H, d, $J=8.0 \mathrm{~Hz}, \mathrm{ArH}), 7.36(2 \mathrm{H}, \mathrm{d}, J=8.4 \mathrm{~Hz}, \mathrm{ArH}), 7.97-8.05(4 \mathrm{H}$, m, ArH $), 8.20(1 \mathrm{H}, \mathrm{s},-\mathrm{OH}), 9.78(1 \mathrm{H}, \mathrm{s},-\mathrm{NH}), 9.99(1 \mathrm{H}, \mathrm{s},-\mathrm{N} H), 13.57(1 \mathrm{H}, \mathrm{s}$, $-\mathrm{OH})$. MS m/z $375.0\left[\mathrm{M}^{+}\right]$

4-Hydroxy- $N$ '-[(2-methoxyphenyl)carbonyl]-2H-1,2-benzothiazine-3carbohydrazide 1,1-dioxide (8d)

Brownish yellow powder; $\mathrm{mp}: 112^{\circ} \mathrm{C}$. IR $(\mathrm{KBr}) \mathrm{Cm}^{-1}: 3751,3654,3564$ $2364,1642,1349,1178,1069 .{ }^{1} \mathrm{H}$ NMR $\left(\mathrm{DMSO}-d_{6}\right)(400 \mathrm{MHz}) \delta: 3.49(1 \mathrm{H}$, $\left.\mathrm{s}, \mathrm{SO}_{2} \mathrm{NH}\right), 3.90\left(3 \mathrm{H}, \mathrm{s},-\mathrm{OCH}_{3}\right), 6.89-6.95(4 \mathrm{H}, \mathrm{m}, \mathrm{ArH}), 7.77-7.90(4 \mathrm{H}, \mathrm{m}$, ArH), $10.18(1 \mathrm{H}, \mathrm{s},-\mathrm{N} H),-10.31(1 \mathrm{H}, \mathrm{s},-\mathrm{N} H), 13.60(1 \mathrm{H}, \mathrm{s},-\mathrm{OH}) . \mathrm{MS} \mathrm{m} / \mathrm{z} 389.3$ $\left[\mathrm{M}^{+}\right]$

4-Hydroxy- $N$ '-[(3-methoxyphenyl)carbonyl]-2H-1,2-benzothiazine-3carbohydrazide 1,1-dioxide (8e)

Yellow powder; mp: $152^{\circ} \mathrm{C}$. IR $(\mathrm{KBr}) \mathrm{Cm}^{-1}: 3754,3653,3564,2364$, $1645,1352,1182,1071 .{ }^{1} \mathrm{H}$ NMR (DMSO- $\left.d_{6}\right)(400 \mathrm{MHz}) \delta: 3.37(1 \mathrm{H}, \mathrm{s}$, $\left.\mathrm{SO}_{2} \mathrm{~N} H\right), 3.93\left(3 \mathrm{H}, \mathrm{s},-\mathrm{OCH}_{3}\right), 6.95(1 \mathrm{H}, \mathrm{d}, J=7.6 \mathrm{~Hz}, \mathrm{ArH}), 7.16(1 \mathrm{H}, \mathrm{d}, J=8.0$ $\mathrm{Hz}, \mathrm{ArH}), 7.26-7.45(4 \mathrm{H}, \mathrm{m}, \mathrm{ArH}), 7.75(1 \mathrm{H}, \mathrm{s}, \mathrm{ArH}), 7.88(1 \mathrm{H}, \mathrm{d}, J=7.2 \mathrm{~Hz}$, ArH $), 9.79(1 \mathrm{H}, \mathrm{s},-\mathrm{N} H), 9.96(1 \mathrm{H}, \mathrm{s},-\mathrm{N} H), 13.61(1 \mathrm{H}, \mathrm{s},-\mathrm{O} H) . \mathrm{MS} \mathrm{m} / \mathrm{z} 389.3\left[\mathrm{M}^{+}\right]$

4-Hydroxy- $N$ '-[(4-methoxyphenyl)carbonyl]-2H-1,2-benzothiazine-3carbohydrazide 1,1-dioxide (8f)

Brownish yellow powder; mp: $119^{\circ} \mathrm{C}$. IR (KBr) $\mathrm{Cm}^{-1}: 3751,3654,3564$, 
2364, 1642, 1349, 1178, 1069. ${ }^{1} \mathrm{H}$ NMR (DMSO- $\left.d_{\text {) }}\right)(400 \mathrm{MHz}) \delta: 3.39(1 \mathrm{H}, \mathrm{s}$, $\left.\mathrm{SO}_{2} \mathrm{NH}\right), 3.91\left(3 \mathrm{H}, \mathrm{s},-\mathrm{OCH}_{3}\right), 6.96(2 \mathrm{H}, \mathrm{d}, J=8.0 \mathrm{~Hz}, \mathrm{ArH}), 7.29(2 \mathrm{H}, \mathrm{d}, J=8.4$ $\mathrm{Hz}, \quad \mathrm{ArH}), \quad 7.90-8.01(4 \mathrm{H}, \mathrm{m}, \mathrm{ArH}), 10.19(1 \mathrm{H}, \mathrm{s},-\mathrm{N} H), 10.32(1 \mathrm{H}, \mathrm{s},-\mathrm{N} H)$, 13.56(1H, s, $-\mathrm{OH}) . \mathrm{MS} \mathrm{m} / \mathrm{z} 389.3\left[\mathrm{M}^{+}\right]$.

$N^{\prime}$-[(2-Bromophenyl)carbonyl]-4-hydroxy-2H-1,2-benzothiazine-3carbohydrazide 1,1-dioxide (8g)

Light yellow powder; mp: $116^{\circ} \mathrm{C}$. IR $(\mathrm{KBr}) \mathrm{Cm}^{-1}: 3751,3654,3564$, 2364, 1642, 1349, 1178, 1069. ${ }^{1} \mathrm{H}$ NMR (DMSO- $\left.d_{6}\right)(400 \mathrm{MHz}) \delta: 3.49(1 \mathrm{H}$, s, $\left.\mathrm{SO}_{2} \mathrm{NH}\right), 6.90-6.95(4 \mathrm{H}, \mathrm{m}, \mathrm{ArH}), \quad 7.78-7.89(4 \mathrm{H}, \mathrm{m}, \mathrm{ArH}), 10.17(1 \mathrm{H}, \mathrm{s},-$ $\mathrm{N} H), 10.30(1 \mathrm{H}, \mathrm{s},-\mathrm{N} H), 13.61(1 \mathrm{H}, \mathrm{s},-\mathrm{O} H) . \mathrm{MS} \mathrm{m} / \mathrm{z} 438\left[\mathrm{M}^{+}\right]$

$N^{\prime}$-[(3-Bromophenyl)carbonyl]-4-hydroxy-2H-1,2-benzothiazine-3carbohydrazide 1,1-dioxide (8h)

Light brown powder; mp: $216^{\circ} \mathrm{C}$. IR $(\mathrm{KBr}) \mathrm{Cm}^{-1}: 3754,3656,3563$, $2365,1643,1348,1180,1072 .{ }^{1} \mathrm{H}$ NMR $\left(\mathrm{DMSO}-d_{6}\right)(400 \mathrm{MH} z) \delta: 3.47(1 \mathrm{H}, \mathrm{s}$, $\left.\mathrm{SO}_{2} \mathrm{~N} H\right), 6.95(1 \mathrm{H}, \mathrm{d}, J=7.6 \mathrm{~Hz}, \mathrm{ArH}), 7.19(1 \mathrm{H}, \mathrm{d}, J=8.0 \mathrm{~Hz}, \mathrm{ArH}), 7.25$ $7.44(4 \mathrm{H}, \mathrm{m}, \mathrm{ArH}), 7.78(1 \mathrm{H}, \mathrm{s}, \mathrm{ArH}), 7.89(1 \mathrm{H}, \mathrm{d}, J=7.2 \mathrm{~Hz}, \mathrm{ArH}), 10.19(1 \mathrm{H}$, $\mathrm{s},-\mathrm{N} H), 10.27(1 \mathrm{H}, \mathrm{s},-\mathrm{N} H), 13.59(1 \mathrm{H}, \mathrm{s},-\mathrm{OH}) . \mathrm{MS} \mathrm{m} / \mathrm{z} 438\left[\mathrm{M}^{+}\right]$

$N^{\prime}$-[(4-Bromophenyl)carbonyl]-4-hydroxy-2H-1,2-benzothiazine-3carbohydrazide 1,1-dioxide (8i)

Light brownish powder; mp: $260^{\circ} \mathrm{C}$. IR $(\mathrm{KBr}) \mathrm{Cm}^{-1}: 3751,3654,3564$, $2364,1642,1349,1178,1069 .{ }^{1} \mathrm{H}$ NMR (DMSO- $\left.d_{6}\right)(400 \mathrm{MH} z) \delta: 3.39(1 \mathrm{H}, \mathrm{s}$, $\left.\mathrm{SO}_{2} \mathrm{NH}\right), 6.96(2 \mathrm{H}, \mathrm{d}, J=8.0 \mathrm{~Hz}, \mathrm{ArH}), 7.30(2 \mathrm{H}, \mathrm{d}, J=8.4 \mathrm{~Hz}, \mathrm{ArH}), 7.92$ $8.02(4 \mathrm{H}, \mathrm{m}, \mathrm{ArH}), 10.19(1 \mathrm{H}, \mathrm{s},-\mathrm{N} H), 10.34(1 \mathrm{H}, \mathrm{s},-\mathrm{N} H), 13.60(1 \mathrm{H}, \mathrm{s},-\mathrm{OH})$

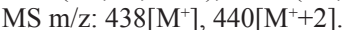

4-Hydroxy- $N$ '-[(2-nitrophenyl)carbonyl]-2H-1,2-benzothiazine-3carbohydrazide 1,1-dioxide (8j)

Pale yellow powder; mp: $155^{\circ} \mathrm{C}$. IR $(\mathrm{KBr}) \mathrm{Cm}^{-1}: 3754,3651,3563$, $2363,1644,1347,1182,1071 .{ }^{1} \mathrm{H}$ NMR (DMSO- $\left.d_{6}\right)(400 \mathrm{MHz}) \delta: 3.50(1 \mathrm{H}, \mathrm{s}$, $\left.\mathrm{SO}_{2} \mathrm{NH}\right), 6.90-6.96(4 \mathrm{H}, \mathrm{m}, \mathrm{ArH}), 7.80-7.91(4 \mathrm{H}, \mathrm{m}, \mathrm{ArH}), 10.16(1 \mathrm{H}, \mathrm{s},-\mathrm{N} H)$, 10.29(1H, s,-NH), 13.62(1H, s, -OH). MS m/z $403.9\left[\mathrm{M}^{+}\right]$

4-Hydroxy- $N$ '-[(3-nitrophenyl)carbonyl]-2H-1,2-benzothiazine-3carbohydrazide 1,1-dioxide (8k)

Yellow powder; $\mathrm{mp}: 230^{\circ} \mathrm{C}$. IR (KBr) $\mathrm{Cm}^{-1}: 3751,3654,3564,2364,1642$, $1349,1178,1069 .{ }^{1} \mathrm{H}$ NMR (DMSO- $\left.d_{6}\right)(400 \mathrm{MHz}) \delta: 3.31\left(1 \mathrm{H}, \mathrm{s}, \mathrm{SO}_{2} \mathrm{~N} H\right)$, 6.98(1H, d, $J=7.6 \mathrm{~Hz}, \operatorname{ArH}), 7.14(1 \mathrm{H}, \mathrm{d}, J=8.0 \mathrm{~Hz}, \operatorname{ArH}), 7.23-7.41^{2}(4 \mathrm{H}$, m, ArH $), 7.79(1 \mathrm{H}, \mathrm{s}, \operatorname{ArH}), 7.88(1 \mathrm{H}, \mathrm{d}, J=7.2 \mathrm{~Hz}, \operatorname{ArH}), 9.77(1 \mathrm{H}, \mathrm{s},-\mathrm{N} H)$, $9.98(1 \mathrm{H}, \mathrm{s},-\mathrm{N} H), 13.59(1 \mathrm{H}, \mathrm{s},-\mathrm{OH}) . \mathrm{MS} \mathrm{m} / \mathrm{z} 403.9\left[\mathrm{M}^{+}\right]$

4-Hydroxy- $N$ '-[(4-nitrophenyl)carbonyl]-2H-1,2-benzothiazine-3carbohydrazide 1,1-dioxide (8I)

Pale yellow powder; $\mathrm{mp}: 155^{\circ} \mathrm{C}$. IR $(\mathrm{KBr}) \mathrm{Cm}^{-1}: 3754,3651,3563$, $2363,1644,1347,1182,1071 .{ }^{1} \mathrm{H}$ NMR (DMSO- $\left.d_{6}\right)(400 \mathrm{MH} z) \delta: 3.49(1 \mathrm{H}, \mathrm{s}$, $\left.\mathrm{SO}_{2} \mathrm{NH}\right), 6.87-6.97(4 \mathrm{H}, \mathrm{m}, \mathrm{ArH}), 7.67-7.82(4 \mathrm{H}, \mathrm{m}, \mathrm{ArH}), 10.17(1 \mathrm{H}, \mathrm{s},-\mathrm{N} H)$ $10.33(1 \mathrm{H}, \mathrm{s},-\mathrm{N} H), 13.62(1 \mathrm{H}, \mathrm{s},-\mathrm{OH}) . \mathrm{MS} \mathrm{m} / \mathrm{z} 403.9\left[\mathrm{M}^{+}\right]$

\section{ANTI-MICROBIAL ACTIVITIES:}

Anti-Bacterial Activity: The anti-bacterial assay was carried out by microdilution method ${ }^{26,27}$ against human pathogenic bacteria. The bacterial suspensions were adjusted with sterile saline to a concentration of $1.0 \times 10^{5}$ colony forming unit $(\mathrm{CFU}) / \mathrm{ml}$. The inocula were prepared daily and stored at $+4^{\circ} \mathrm{C}$ until use. Dilutions of the inocula were cultured on solid medium to verify the absence of contamination and to check the validity of the inoculum. The minimum inhibitory concentrations (MICs) were determined using 96well microtitre plates. Compounds under investigation were dissolved in broth LB medium $(100 \mu \mathrm{l})$ with bacterial inoculum $\left(1.0 \times 10^{4} \mathrm{cfu}\right.$ per well $)$ to achieve the desired concentrations $(1 \mathrm{mg} / \mathrm{ml})$. All the microplates were incubated for 24 hours at $48^{\circ} \mathrm{C}$. The optical density of each well was measured at a wavelength of $655 \mathrm{~nm}$ and compared with a blank. Streptomycin was used as a positive control (1 mg/ ml DMSO).

Anti-fungal Activity: Anti-fungal screening of the newly synthesized compounds was carried out by micro dilution technique ${ }^{26}$ against Aspergillus flavus, Trichoderma reesei, Drechslera australiensis, Trichoderma viride, Alternaria alternate and Macrophomina phaseolina. The micromycetes were maintained on malt agar and the cultures were stored at $4^{\circ} \mathrm{C}$ and sub-cultured once a month ${ }^{28}$. Fungal spores were washed from the surface of agar plates with sterile $0.85 \%$ saline containing $0.1 \%$ Tween $80(\mathrm{v} / \mathrm{v})$ and the spore suspension was adjusted with sterile saline to a concentration of approximately $1.0 \times 10^{5}$ in a final volume of $100 \mu \mathrm{l}$ per well. Minimum inhibitory concentration (MIC) determinations were performed by a serial dilution technique using 96 -well microtiter plates. The compounds investigated were dissolved in DMSO $(1 \mathrm{mg} /$ $\mathrm{ml}$ ) and were added in broth malt medium with inoculum. The microplates were incubated for 72 hours at $28^{\circ} \mathrm{C}$. The lowest concentrations without visible growth (at the binocular microscope) were defined as MICs.

\section{RESULTS AND DISCUSSION}

During the course of the work, using the green innovative technology ethyl (1,1-dioxido-3-oxo-1,2-benzisothiazol-2(3H)-yl)- acetate (3) was synthesized by the reaction of sodium saccharin (1) and ethyl chloroacetate (2) followed by its ring expansion to six membered benzothiazine through Gabriel-Coleman type rearrangement yielding ethyl 4-hydroxy-2H-1,2-benzothiazine-3carboxylate 1,1-dioxide (4). The formation of ethyl (1,1-dioxido-3-oxo-1,2benzisothiazol-2(3H)-yl)- acetate (3) was carried out on water bath and the formation of ethyl 4-hydroxy-2H-1,2-benzothiazine-3-carboxylate 1,1-dioxide (4) was carried out using ultrasonic waves.

Synthesis of alkyl benzoates (6a-1), substituted benzohydrazides (7a-l) and 4-hydroxy- $N$ '-(phenylcarbonyl)-2H-1,2-benzothiazine-3-carbohydrazide 1,1-dioxides compounds (8a-l) were synthesized as per scheme 1 through environment friendly fashion by the use of microwaves. Alkyl benzoate derivatives (6a-l), were synthesized by acid catalysis of respective benzoic acid under microwaves in excellent yields $(92-97 \%$; Table 1) which were converted to the corresponding benzohydrazide derivatives (7a-I) by microwave induced hydrazinolyses in very good to excellent yields (81-96\%). These benzohydrazides were then reacted with ethyl 4-hydroxy- $2 H-1,2-$ benzothiazine-3-carboxylate 1,1-dioxide (4) to get a series of 4-hydroxy- $N^{\prime}$ (phenylcarbonyl)-2H-1,2-benzothiazine-3-carbohydrazide 1,1-dioxides (8al). These reactions were carried out under the influence of microwaves and were completed in good yields with in shorter reaction times (Table 1). All of the newly synthesized compounds were characterized through spectroscopic techniques and their data was found in accordance to the proposed structures.

\section{Anti-bacterial activity}

All the newly synthesized carbohydrazide derivatives (dissolved in dimethylformamide) were subjected to anti-bacterial screening against three gram positive bacteria (Bacillus cereus, Bacillus subtilis and Bacillus thuringiensis) and three gram negative bacteria (Escherichia coli, Pseudomonas aeruginosa, Salmonella typhi) by determining their minimum inhibitory concentrations (MICs) using agar dilution technique ${ }^{29}$ and streptomycin as a reference drug. The choice of streptomycin as a clinical standard was made due to the fact that at lower concentrations, it only inhibits growth of the bacteria through induction of prokaryotic ribosomes to misread mRNA. It also prevents initiation of protein synthesis and leads to death of microbial cells. Also, in humans, they have structurally different ribosomes from bacteria, thereby allowing the selectivity of this antibiotic for bacteria ${ }^{27}$

The Minimum inhibitory concentrations of the synthesized compounds against selected bacteria are presented in Table 2. Results of anti-bacterial activity of compounds show that the minimum inhibitory concentration (MIC) of compounds varies in the range of $6.0-67.0 \times 10^{-2} \mu \mathrm{mol} / \mathrm{ml}$. It was found that the title compounds were found more active against gram positive bacteria than gram negative bacteria. The most sensitive bacterial species on these compounds is Bacillus subtilis, while Pseudomonas aeruginosa is the most resistant species. An insight to the structure-activity relationship gives an idea that activity generally increases with the incorporation of hydroxyl and methoxy groups at the benzene ring of the title compounds. Hydroxy derivatives $(\mathbf{8 a - 8 c})$ were found the most active with MIC values comparable to the standard and this may be attributed to the presence of lone pairs on oxygen atoms of hydroxyl groups perhaps by blocking the active sites through hydrogen bonding. Compounds having methoxy groups are found weaker than the hydroxyl analogues probably due to steric hindrance of methyl group. Compounds possessing nitro groups $(\mathbf{8 j - 8})$ were found least active against both the gram positive and gram negative strains. 
Table 1. Data of the synthesized compounds (6a- 81).

\begin{tabular}{|c|c|c|c|c|c|c|c|}
\hline Entry & Compound & $\mathbf{R}_{1}$ & $\mathbf{R}_{2}$ & $\mathbf{R}_{3}$ & M.P/ B.P & $\begin{array}{c}\text { Reaction } \\
\text { time }\end{array}$ & Yield \\
\hline & $6 a$ & $\mathrm{OH}$ & $\mathrm{H}$ & $\mathrm{H}$ & $224^{\circ} \mathrm{C}^{*}$ & 17 minutes & $94 \%$ \\
\hline & $6 \mathrm{~b}$ & $\mathrm{H}$ & $\mathrm{OH}$ & $\mathrm{H}$ & $70^{\circ} \mathrm{C}$ & 19 minutes & $93 \%$ \\
\hline & $6 \mathrm{c}$ & $\mathrm{H}$ & $\mathrm{H}$ & $\mathrm{OH}$ & $126^{\circ} \mathrm{C}$ & 13 minutes & $96 \%$ \\
\hline & $6 \mathrm{~d}$ & $\mathrm{OCH}_{3}$ & $\mathrm{H}$ & $\mathrm{H}$ & $247^{\circ} \mathrm{C}^{*}$ & 18 minutes & $94 \%$ \\
\hline & $6 \mathrm{e}$ & $\mathrm{H}$ & $\mathrm{OCH}_{3}$ & $\mathrm{H}$ & $238^{\circ} \mathrm{C}^{*}$ & 20 minutes & $95 \%$ \\
\hline & $6 f$ & $\mathrm{H}$ & $\mathrm{H}$ & $\mathrm{OCH}_{3}$ & $50^{\circ} \mathrm{C}$ & 15 minutes & $96 \%$ \\
\hline & $6 \mathrm{~g}$ & $\mathrm{Br}$ & $\mathrm{H}$ & $\mathrm{H}$ & $241^{\circ} \mathrm{C}^{*}$ & 15 minutes & $95 \%$ \\
\hline & $6 \mathrm{~h}$ & $\mathrm{H}$ & $-\mathrm{Br}$ & $\mathrm{H}$ & $140^{\circ} \mathrm{C}^{*}$ & 18 minutes & $94 \%$ \\
\hline & $6 \mathrm{i}$ & $\mathrm{H}$ & $\mathrm{H}$ & $\mathrm{Br}$ & $78^{\circ} \mathrm{C}$ & 12 minutes & $96 \%$ \\
\hline & $6 \mathrm{j}$ & $\mathrm{NO}_{2}$ & $\mathrm{H}$ & $\mathrm{H}$ & $275^{\circ} \mathrm{C}^{*}$ & 19 minutes & $92 \%$ \\
\hline & $6 \mathrm{k}$ & $\mathrm{H}$ & $\mathrm{NO}_{2}$ & $\mathrm{H}$ & $78^{\circ} \mathrm{C}$ & 14 minutes & $97 \%$ \\
\hline & 61 & $\mathrm{H}$ & $\mathrm{H}$ & $\mathrm{NO}_{2}$ & $96^{\circ} \mathrm{C}$ & 16 minutes & $95 \%$ \\
\hline & $7 \mathrm{a}$ & $\mathrm{OH}$ & $\mathrm{H}$ & $\mathrm{H}$ & $148^{\circ} \mathrm{C}$ & 10 minutes & $85 \%$ \\
\hline & $7 \mathrm{~b}$ & $\mathrm{H}$ & $\mathrm{OH}$ & $\mathrm{H}$ & $88^{\circ} \mathrm{C}$ & 15 minutes & $81 \%$ \\
\hline & $7 \mathrm{c}$ & $\mathrm{H}$ & $\mathrm{H}$ & $\mathrm{OH}$ & $266^{\circ} \mathrm{C}$ dec. & 12 minutes & $93 \%$ \\
\hline & $7 d$ & $\mathrm{OCH}_{3}$ & $\mathrm{H}$ & $\mathrm{H}$ & $78^{\circ} \mathrm{C}$ & 13 minutes & $87 \%$ \\
\hline & $7 \mathrm{e}$ & $\mathrm{H}$ & $\mathrm{OCH}_{3}$ & $\mathrm{H}$ & $92^{\circ} \mathrm{C}$ & 16 minutes & $86 \%$ \\
\hline & $7 f$ & $\mathrm{H}$ & $\mathrm{H}$ & $\mathrm{OCH}_{3}$ & $137^{\circ} \mathrm{C}$ & 11 minutes & $96 \%$ \\
\hline & $7 \mathrm{~g}$ & $\mathrm{Br}$ & $\mathrm{H}$ & $\mathrm{H}$ & $152^{\circ} \mathrm{C}$ & 14 minutes & $89 \%$ \\
\hline & $7 \mathrm{~h}$ & $\mathrm{H}$ & $\mathrm{Br}$ & $\mathrm{H}$ & $155^{\circ} \mathrm{C}$ & 15 minutes & $83 \%$ \\
\hline & $7 \mathrm{i}$ & $\mathrm{H}$ & $\mathrm{H}$ & $\mathrm{Br}$ & $168^{\circ} \mathrm{C}$ & 14 minutes & $91 \%$ \\
\hline & $7 \mathrm{j}$ & $\mathrm{NO}_{2}$ & $\mathrm{H}$ & $\mathrm{H}$ & $120^{\circ} \mathrm{C}$ & 18 minutes & $88 \%$ \\
\hline & $7 \mathrm{k}$ & $\mathrm{H}$ & $\mathrm{NO}_{2}$ & $\mathrm{H}$ & $152^{\circ} \mathrm{C}$ & 12 minutes & $95 \%$ \\
\hline & 71 & $\mathrm{H}$ & $\mathrm{H}$ & $\mathrm{NO}_{2}$ & $215^{\circ} \mathrm{C}$ & 15 minutes & $91 \%$ \\
\hline & $8 \mathrm{a}$ & $\mathrm{OH}$ & $\mathrm{H}$ & $\mathrm{H}$ & $116^{\circ} \mathrm{C}$ & 21 minutes & $81 \%$ \\
\hline & $8 \mathrm{~b}$ & $\mathrm{H}$ & $\mathrm{OH}$ & $\mathrm{H}$ & $150^{\circ} \mathrm{C}$ & 23 minutes & $79 \%$ \\
\hline & $8 \mathrm{c}$ & $\mathrm{H}$ & $\mathrm{H}$ & $\mathrm{OH}$ & $185^{\circ} \mathrm{C}$ & 17 minutes & $84 \%$ \\
\hline & $8 \mathrm{~d}$ & $\mathrm{OCH}_{3}$ & $\mathrm{H}$ & $\mathrm{H}$ & $112^{\circ} \mathrm{C}$ & 15 minutes & $81 \%$ \\
\hline & $8 \mathrm{e}$ & $\mathrm{H}$ & $\mathrm{OCH}_{3}$ & $\mathrm{H}$ & $152^{\circ} \mathrm{C}$ & 18 minutes & $77 \%$ \\
\hline & $8 \mathrm{f}$ & $\mathrm{H}$ & $\mathrm{H}$ & $\mathrm{OCH}_{3}$ & $119^{\circ} \mathrm{C}$ & 12 minutes & $85 \%$ \\
\hline & $8 \mathrm{~g}$ & $\mathrm{Br}$ & $\mathrm{H}$ & $\mathrm{H}$ & $116^{\circ} \mathrm{C}$ & 20 minutes & $75 \%$ \\
\hline & $8 \mathrm{~h}$ & $\mathrm{H}$ & $\mathrm{Br}$ & $\mathrm{H}$ & $216^{\circ} \mathrm{C}$ & 25 minutes & $73 \%$ \\
\hline & $8 \mathrm{i}$ & $\mathrm{H}$ & $\mathrm{H}$ & $\mathrm{Br}$ & $260^{\circ} \mathrm{C}$ & 15 minutes & $80 \%$ \\
\hline & $8 \mathrm{j}$ & $\mathrm{NO}_{2}$ & $\mathrm{H}$ & $\mathrm{H}$ & $155^{\circ} \mathrm{C}$ & 22 minutes & $82 \%$ \\
\hline & $8 \mathrm{k}$ & $\mathrm{H}$ & $\mathrm{NO}_{2}$ & $\mathrm{H}$ & $230^{\circ} \mathrm{C}$ & 14 minutes & $86 \%$ \\
\hline & 81 & $\mathrm{H}$ & $\mathrm{H}$ & $\mathrm{NO}_{2}$ & $155^{\circ} \mathrm{C}$ & 20 minutes & $74 \%$ \\
\hline
\end{tabular}

* = Boiling point

Dec. $=$ Decomposition

Table 2. Anti-bacterial activity of compounds (8a-1) tested by micro dilution method (MIC $\mu$ mol x 10-2).

\begin{tabular}{|c|c|c|c|c|c|c|c|}
\hline \multirow{2}{*}{ Entry } & \multirow{2}{*}{ Compound } & \multicolumn{6}{|c|}{ Susceptible Bacteria } \\
\hline & & E. coli & P. aeruginosa & S. typhi & B. cereus & B. subtilis & B. thuringiensis \\
\hline 1 & $8 a$ & 25 & 26 & 22 & 9 & 8 & 22 \\
\hline 2 & $8 \mathbf{b}$ & 21 & 23 & 21 & 11 & 6 & 20 \\
\hline 3 & $8 c$ & 30 & 28 & 24 & 14 & 9 & 23 \\
\hline 4 & $8 d$ & 39 & 35 & 38 & 17 & 14 & 20 \\
\hline 5 & $8 \mathrm{e}$ & 37 & 31 & 33 & 16 & 18 & 19 \\
\hline 6 & $8 f$ & 40 & 46 & 42 & 19 & 15 & 21 \\
\hline 7 & $8 g$ & 60 & 56 & 55 & 22 & 22 & 25 \\
\hline 8 & $8 \mathrm{~h}$ & 66 & 59 & 51 & 18 & 24 & 23 \\
\hline 9 & $8 \mathbf{i}$ & 61 & 67 & 49 & 20 & 20 & 27 \\
\hline 10 & $8 \mathbf{j}$ & 58 & 44 & 48 & 20 & 17 & 18 \\
\hline 11 & $8 k$ & 59 & 49 & 42 & 20 & 19 & 20 \\
\hline 12 & 81 & 63 & 51 & 52 & 23 & 24 & 18 \\
\hline 13 & Control & 0 & 0 & 0 & 0 & 0 & 0 \\
\hline 14 & Streptomycin & 16.5 & 16.5 & 16.5 & 4.3 & 4.2 & 4.4 \\
\hline
\end{tabular}


Anti-fungal activities

All the newly synthesized compounds 8a-8I (dissolved in dimethylsulfoxide) along with ethyl 4-hydroxy-2H-1,2-benzothiazine-3-carboxylate 1,1-dioxide (4), bifonazole (reference drug) and control $\mathbf{0}$ were subjected to in-vitro anti-fungal screening by determining the minimum inhibitory concentration (MICs) through micro dilution technique ${ }^{29}$ against a panel of six strains of fungi i.e., Aspergillus flavus, Trichoderma reesei, Drechslera australiensis, Trichoderma viride, Alternaria alternate and Macrophomina phaseolina.

Results of anti-fungal activity of compounds show that the minimum inhibitory concentration (MIC) of compounds varies in the range of $68.1-124.0 \times 10^{-2}$ $\mu \mathrm{mol} / \mathrm{ml}$ [Table 3]. Compounds $\mathbf{8 j}, \mathbf{8 k}$ and $\mathbf{8} \mathbf{l}$ showed the best activity against all the fungi, with $\mathbf{8 i}$ exhibiting the highest antifungal potential (MIC $68.1 \times 10^{-2}$ $\mu \mathrm{mol} / \mathrm{ml}$, while compounds $\mathbf{8 g}, \mathbf{8} \mathbf{h}$ and $8 \mathbf{i}$ were found moderately active. Rest of the compounds were found almost inactive (with $\mathrm{MIC}>100 \mathrm{x} 10^{-2} \mu \mathrm{mol} / \mathrm{ml}$ ).

Table 3. Anti-fungal activity of compounds (8a-1) tested by micro dilution method (MIC $\mu \mathrm{mol} \times 10^{-2}$ ).

\begin{tabular}{|c|c|c|c|c|c|c|c|}
\hline \multirow[b]{2}{*}{ Entry } & \multirow[b]{2}{*}{ Compound } & \multicolumn{6}{|c|}{ Susceptible Fungi } \\
\hline & & $\begin{array}{l}\text { Aspergillus } \\
\text { flavus }\end{array}$ & $\begin{array}{c}\text { Trichoderma } \\
\text { reesei }\end{array}$ & $\begin{array}{c}\text { Drechslera } \\
\text { australiensis }\end{array}$ & $\begin{array}{c}\text { Trichoderma } \\
\text { viride }\end{array}$ & $\begin{array}{c}\text { Alternaria } \\
\text { alternata }\end{array}$ & $\begin{array}{l}\text { Macrophomina } \\
\text { phaseolina }\end{array}$ \\
\hline 1 & 8a & $>100$ & 98.0 & $>100$ & 97.3 & 98.0 & 96.5 \\
\hline 2 & $8 \mathbf{b}$ & $>100$ & 96.5 & $>100$ & 94.2 & $>100$ & 95.2 \\
\hline 3 & $8 \mathbf{c}$ & $>100$ & 99.6 & $>100$ & 97.9 & $>100$ & $>100$ \\
\hline 4 & 8d & $>100$ & 81.3 & $>100$ & 98.8 & $>100$ & $>100$ \\
\hline 5 & $8 \mathbf{8}$ & $>100$ & 80.6 & 92.8 & 97.6 & $>100$ & $>100$ \\
\hline 6 & $8 f$ & $>100$ & 79.9 & 89.9 & 95.1 & 93.3 & 96.2 \\
\hline 7 & $8 g$ & $>100$ & 77.4 & 99.7 & 75.6 & 81.5 & 87.4 \\
\hline 8 & $\mathbf{8 h}$ & $>100$ & 74.9 & 98.8 & 74.4 & 78.9 & 85.6 \\
\hline 9 & $8 \mathbf{i}$ & $>100$ & 72.7 & 95.4 & 71.6 & 77.3 & 83.9 \\
\hline 10 & $8 \mathrm{j}$ & $>100$ & 72.5 & 92.0 & 71.9 & 80.1 & 82.6 \\
\hline 11 & $8 \mathbf{k}$ & $>100$ & 70.1 & 90.9 & 69.6 & 77.8 & 81.2 \\
\hline 12 & 81 & $>100$ & 68.4 & 87.0 & 68.1 & 74.8 & 78.3 \\
\hline 13 & $\mathbf{0}$ & 0 & 0 & 0 & 0 & 0 & 0 \\
\hline 14 & 4 & $>100$ & $>100$ & $>100$ & $>100$ & $>100$ & $>100$ \\
\hline 15 & Bifonazole & 48.0 & 60.2 & 57.5 & 64.0 & 58.0 & 58.0 \\
\hline
\end{tabular}

Results showed moderate to significant activity of almost all the compounds against Trichoderma reesei, Drechslera australiensis, Trichoderma viride, Alternaria alternate and Macrophomina phaseolina while these were found inactive against Aspergillus flavus; all of the compounds were either found inactive or with MIC greater than 100. Majority of the compounds showed good activity against Trichoderma viride and Trichoderma reesei while Aspergillus flavus is the most resistant species. An insight to the structureactivity relationship gives an idea that activity generally increases with substitution groups having electron withdrawing nature.

\section{CONCLUSION}

The present study revealed that the compounds obtained by synergism of 4-hydroxy-2H-1,2-benzothiazine-3-carboxylate 1,1-dioxide moiety with different carbohydrazides in one frame were found to possess anti-bacterial \& anti-fungal activities and could be useful as a template for future development through modification or derivatization to design more potent biologically active compounds. The new skeleton may also possess other biological activities of the parent ring systems, if tested.

\section{REFERENCES}

1. A. R. Katritzky, D. A. Nichols, M. Siskin, R. Murgan, M. Belasubramanian, Chem. Rev., 101, 837, (2001).

2. M. Siskin, A. R. Katritzky, Chem. Rev., 101, 825, (2001).

3. F. G. Klarner, V. Breitkopf, Eur. J. Org. Chem., 27, 57, (1999).

4. P. G. Jessop, T. Ikariya, R. Noyori, Chem. Rev., 99, 475, (1999).

5. M. J. Earle, P. B. Mccormac, K. R. Seddon, Green Chem., 1, 23, (1999).

6. H. Krister, Adv. Colloid Intarface Sci., 51, 137, (1994).

7. A. Fini, A. Breccia, Pure. Appl. Chem., 71, 573, (1999).

8. L. Perreux, A. Loupy, Tetrahedron, 57, 9199, (2001).

9. S. Polanc, Targets Heterocycl. Syst., 3, 33, (1999).

10. M. M. Dutta, B. N. Goswami, J. C. S. Kataky, J. Heterocyclic Chem. 23 793, (1986).

11. P. H. Erman, H. Straub, U.S. Pat. US 5,318,963, Jun 7, (1994).

12. L.Troeberg, X. Chen, T. M. Flaherty, R. E. Morty, M. Cheng, H. Hua, C. Springer, J. H. Mc Kerrow, G. L. Kenyon, J. D. Lonsdale-Eccles, T. H. T. Coetzer, F. E. Cohen, Mol. Med. (N.Y.), 6, 660, (2000).
13. H. L.Yale, K. Losee, J. Martins, M. Holsing, F. M. Perry, J. Bernstein, J. Am. Chem. Soc. 75, 1933, (1953).

14. T. R. Opie, Eur. Pat. Appl. EP 984,009, Mar 8, (2000).

15. B. Silvestrini, C. Y. Cheng, U.S. Pat. US 6,001,865 Dec. 14, (1999).

16. N. Ahmed, M. Zia-ur-Rehman, H.L. Siddiqui, M. Fasih Ullah, M. Parvez, Eur, J. Med. Chem, 46, 2368,m (2011).

17. J. Braun, Chem. Ber., 56, 2332, (1923).

18. D. Turck, U. Busch, G. Heinzel, H. Narjes, G. Nehmiz, J. Clin. Pharmacol. 36, 79, (1996).

19. B. Cottineau, P. Toto, C. Marot, A. Pipaud, J. Chenault, Bioorg. Med. Chem. Lett. 12, 2105, (2002).

20. S.R. Smith, G. Denhardt, C. Terminelli, Eur. J. Pharmacol. 432, 107, (2001).

21. M. Zia-ur-Rehman, J. A. Choudary, M.R.J. Elsegood, H. L. Siddiqui, K. M. Khan, Eur. J. Med. Chem. 44, 1311, (2009).

22. M. N. Arshad, I. U. Khan, M. Zia-ur-Rehman, M. Shafiq, Asian J. Chem. 23, 2801, (2011).

23. M. Shafiq, I.U.Khan, M. Zia-ur-Rehman, A. M. Asiri, M. N. Arshad, Asian J. Chem. 24, 4799, (2012).

24. M. Ahmad, H. L. Siddiqui, M. Zia-ur-Rehman, M. Parvez, Eur. J. Med. Chem. 45, 698 (2010).

25. C. Gennari, B. Salom, D. Potenza, A. Williams, Angew. Chem. Int. Ed. Engl. 33, 2067, (1994).

26. H. Hanel, W. Raether, Mycoses, 31, 148, (1988).

27. J. G. Colle, J. P. Duguid, A. G. Fraser, B. P. Marmion. Practical Medical Biology, ed. by T.J. Mackie, J.E. McCartney, thirteenth ed. Churchill Livingstone, London, UK, 1989

28. C. Booth Methods in Microbiology Vol. IV, ed. by J. R. Norris, D. W. Ribbons, Academic Press, London, New York, pp. 49-94, (1971).

29. A. Espinel-Ingroff, J. Clin. Microbiol., 39, 1360, (2001). 